\title{
$\mathrm{PH} \mathbf{8} \mathbf{7}_{\text {bienes, paisajes e itinerarios }}$
}

\section{El acueducto romano de Almuñécar}

\author{
Elena Sánchez López | Universidad de Granada \\ URL de la contribución <www.iaph.es/revistaph/index.php/revistaph/article/view/3572>
}

\section{RESUMEN}

La antigua ciudad de Sexi Firmum Iulium se dotó en el siglo I d. C., coincidiendo con su cambio de estatus político-administrativo, de un complejo sistema para garantizar su abastecimiento de agua. Con una longitud total de entre 7 y 10 kilómetros, el acueducto romano de Almuñécar contó con tramos en túnel, canales subterráneos y sectores elevados sobre arcos (arcuatios), además de un complejo sistema de sifón inverso que constituye el último tramo de la conducción antes de su entrada en la ciudad.

Para facilitar su estudio y presentación, las investigaciones recientes sobre el acueducto han dividido su trazado en cinco sectores diferentes, que han sido respetados en este escrito. El primero se corresponde con la captación; el segundo con un tramo en el que no existen restos de adscripción romana. Los tramos tercero y cuarto se corresponden con los sectores mejor conocidos, en las cuencas de los ríos Verde y Seco respectivamente. El quinto tramo es el ya mencionado sifón terminal.

\section{Palabras clave}

Abastecimiento de agua | Acueductos | Almuñécar (Granada) | Arqueología | Costa de Granada | Época romana | 


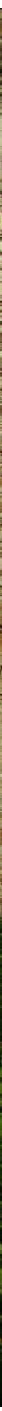

Acueducto romano de Almuñécar | foto Enrique Navarro 
Trazado del acueducto de Almuñécar y los tramos diferenciados | mapa Elena Sánchez López

2. Dibujo de la galería de Las Angosturas | dibujo José M. ${ }^{a}$ de Sancha

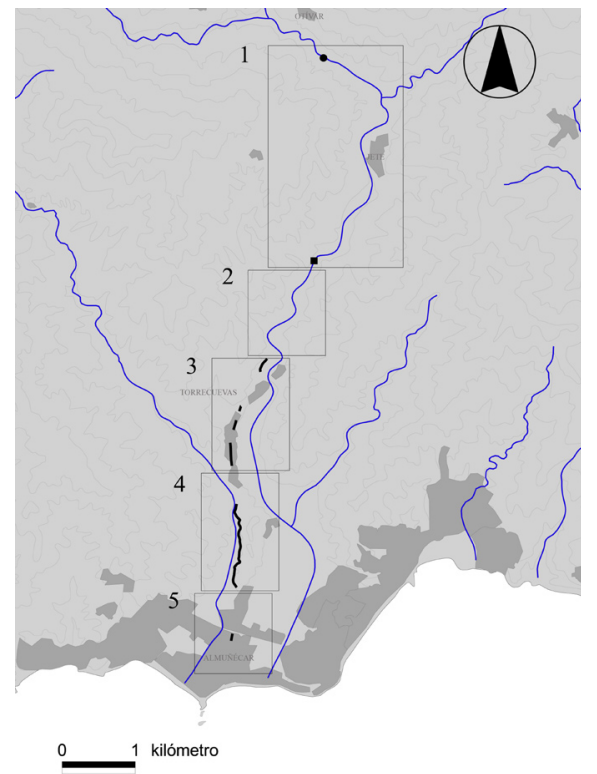

La ciudad romana de Sexi Firmum Iulium, bajo el solar de la actual Almuñécar, fue abastecida por un acueducto construido en la primera mitad del siglo I d. C., poco después de que la ciudad se integrase en el organigrama romano con el estatuto de municipium, y coincidiendo con un momento de gran desarrollo económico y constructivo (SÁNCHEZ LÓPEZ; PÉREZ MARRERO; ORFILA PONS et ál., 2010; SÁNCHEZ LÓPEZ; MORENO PÉREZ, 2012).

El acueducto contaba con un recorrido de al menos $7 \mathrm{~km}$, aunque las investigaciones más recientes han propuesto un trazado de hasta $10 \mathrm{~km}$; corriendo paralelo primero a la margen occidental de la cuenca de río Verde y aguas abajo a la oriental de río Seco. Para facilitar su estudio, se ha procedido a su fragmentación en cinco tramos diferentes (imagen 1) (SÁNCHEZ LÓPEZ, 2011; 2014; SÁNCHEZ LÓPEZ; PÉREZ MARRERO; ORFILA PONS et ál., 2009; 2010; SÁNCHEZ LÓPEZ; MORENO PÉREZ, 2012)

\section{Primer tramo}

Según los primeros estudios realizados sobre el acueducto (FERNÁNDEZ CASADO, 1949; 2008; MOLINA FAJARDO, 2000), la captación de aguas se localiza en la zona de Las Angosturas (TM de Jete), lugar donde en 1875 se encontró una galería de infiltración bajo el lecho del río Verde. Gracias a un dibujo realizado ese mismo año por el ingeniero José María de Sancha (imagen 2), se sabe que la estructura presenta una planta en forma de T. La obra, realizada en obra seca de lajas de piedra y cantos de río, garantizaba

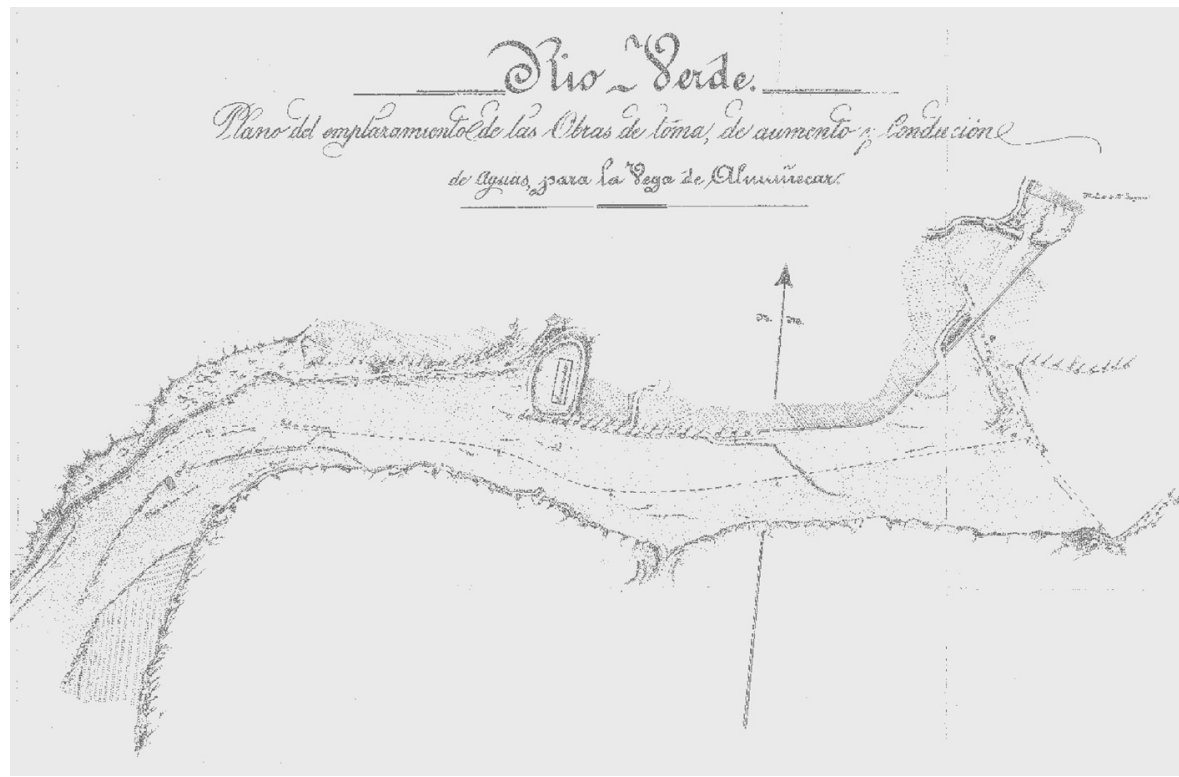


la infiltración por su parte superior, presentando revestimiento interno únicamente en la parte inferior del specus. Hoy en día, sin embargo, no está clara la adscripción romana de esta galería.

En 1992, durante las obras del encauzamiento de río Verde, fue localizada, aguas arriba del pueblo de Jete, un tramo de canal con una tipología y un sistema constructivo similar a los restos identificados a lo largo del recorrido conocido del acueducto; circunstancia que llevó a proponer el inicio de la conducción destinada a la captación de aguas para Almuñécar en las cercanías de Otívar (YÁÑEZ JERÓNIMO; GONZÁLEZ ALMENDROS; BUSTOS PRETEL, 1994; SÁNCHEZ LÓPEZ, 2011), probablemente en el paraje denominado Peñón Rodado. Hasta el momento no se ha podido esclarecer la relación que existió entre estos dos elementos.

\section{Segundo tramo}

El agua sale a la superficie en la conocida como Fuente de los Granados, discurriendo por la denominada Acequia del Rey, una construcción moderna de 1,5 km de longitud. En este sector no existe constancia de restos de la canalización romana.

\section{Tercer tramo}

El canal del acueducto aparece actualmente algo al norte del actual puente de la Autovía A7 que cruza río Verde, coincidiendo con el inicio de un sector excavado en la roca (imagen 3). Tras el túnel, de aproximadamente $30 \mathrm{~m}$ de longitud, el canal es visible semisoterrado durante unos $200 \mathrm{~m}$ presentando la fábrica que se mantendrá a lo largo del resto de su trazado: un canal abovedado realizado en opus incertum de lajas de pizarra trabadas con mortero y con revestimiento interior.

La conducción reaparece tras cruzar la Carretera del Suspiro del Moro, donde atraviesa el barranco de Torrecuevas mediante una construcción de $130 \mathrm{~m}$ de longitud. En la actualidad, este tramo del canal ha sido profundamente transformado, fruto de una dudosa restauración que convirtió en plana una cubierta que, como sucede en los siguientes tramos sobre arcuatio conservados, debió ser abovedada.

Después de cruzar de nuevo la carretera, el canal discurre soterrado a través de tierras de cultivo y bajo construcciones recientes hasta reaparecer elevado sobre un pequeño arco moderno, reconstrucción de uno antiguo que formaba parte de una construcción con al menos dos arcos. A unos $100 \mathrm{~m}$ de esta arcuatio existió otra formada por tres arcos, que actualmente se encuentra totalmente enterrada bajo tierras de cultivo, siendo visibles solamente el canal y la clave de uno de los arcos.

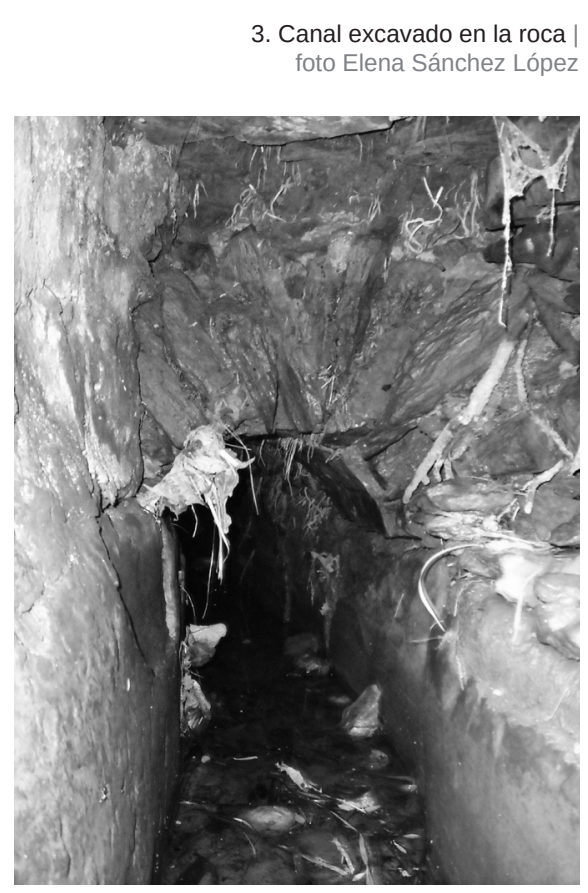




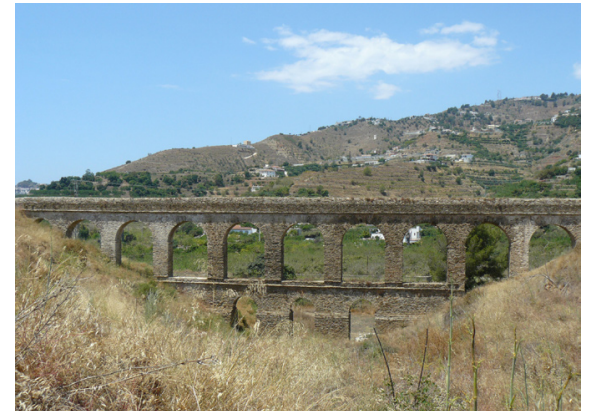

4. Acueducto III | foto Elena Sánchez López

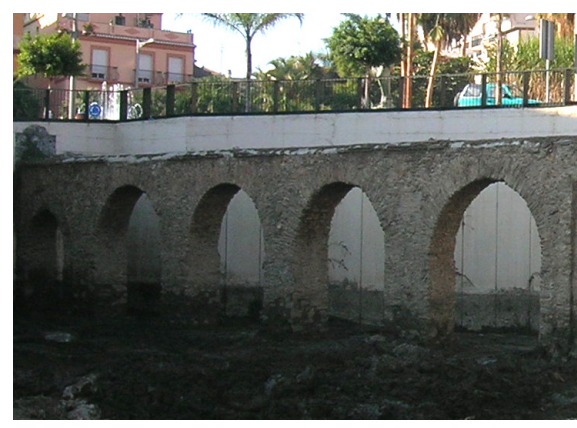

5. Venter del sifón en La Carrera | foto Elena Sánchez López
La canalización reaparece a unos 200 m elevada sobre una arcuatio de cuatro arcos bastante deteriorada debido al corrimiento de tierras sufrido por la terraza de cultivo a la que sirve de apoyo.

A $60 \mathrm{~m}$ aguas abajo aparece el primer spiramen conservado, elemento que constituye asimismo el último punto donde se identifican restos del acueducto en el valle de río Verde, y es que a partir de este momento pasa a discurrir por entero bajo edificaciones modernas, antes de realizar el cambio de cuenca mediante un túnel parcialmente descrito por Fernández Casado (1949) y Tovar Sabio y Camero Ucles (1986).

\section{Cuarto tramo}

El canal del acueducto vuelve a aparecer en la cuenca de río Seco en diferentes puntos, antes de emerger en un breve tramo de canal abovedado, que desemboca en un registro cuadrangular que funcionó como desarenador. A lo largo del trazado de la canalización por este valle, estos elementos van siempre asociados a la existencia de una construcción sobre arcadas, como demuestra la presencia de una moldura similar a la observable en los denominados acueductos I, II y III, así como la clave de un arco emergiendo a pocos centímetros del actual nivel de la terraza de cultivo. A $100 \mathrm{~m}$ de aquí aparece un nuevo registro circular. A partir de este punto, el canal aparece visible a lo largo de un kilómetro, empleado como apoyo para la formación de terrazas de cultivo. Un recorrido a lo largo del cual el canal aparecerá jalonado por multitud de registros y elevado en tres ocasiones sobre sendas arcuatios.

$>$ Acueducto I: una arcuatio de $43 \mathrm{~m}$ de longitud, formada por seis arcos.

$>$ Acueducto II: de $68 \mathrm{~m}$ de longitud. Construida para salvar una vaguada de dimensiones mayores que la anterior, esta arcuatio cuenta con dos alturas de arcos, concretamente tres arcos secundarios bajo los tres centrales.

$>$ Acueducto III (imagen 4): con 72 m de longitud, está formado por dos cuerpos independientes de arcadas. El superior consta de once arcos; el inferior consiste en un basamento con tres huecos rematados por arcos que coinciden con los tres arcos centrales.

\section{Quinto tramo}

A unas decenas de metros del Acueducto III debió localizarse el depósito de entrada del sifón que permitía elevar el agua hasta la ciudad, situada en el Cerro de San Miguel. De este sistema el único elemento visible en la actualidad lo constituyen los diecisiete arcos excavados en la zona de La Carrera, construidos como parte del venter del sifón (JOYANES PÉREZ, 1987; MOLINA FAJARDO, 2000) y destinados a sostener el canal que contenía la canalización cerámica a presión (imagen 5). 


\section{BIBLIOGRAFÍA}

- FERNÁNDEZ CASADO, C. (1949) La conducción romana de aguas de Almuñécar. Archivo Español de Arqueología, n. ${ }^{\circ} 22,1949$, pp. 313-333

- FERNÁNDEZ CASADO, C. (2008) La conducción romana de aguas de Almuñécar. Acueductos romanos en España, vol. 2. Madrid: Colegio de Ingenieros de Caminos Canales y Puertos, 2008, pp. 147-161

- JOYANES PÉREZ, M. (1987) Prospección arqueológica con sondeos estratigráficos en el tramo IV del acueducto romano de Almuñecar (Granada). Anuario Arqueológico de Andalucía 1986, II, 1987, pp. 232-235

- MOlinA FAJARDo, F. (2000) El Acueducto. En MOLINA FAJARDO, F. Almuñécar romana. Almuñécar: Ayuntamiento, 2000, pp. 87-111

- SÁNCHEZ LÓPEZ, E. (2011) AQUA SEXITANA. La relación del acueducto romano de Almuñécar con Sexi Firmum Iulium y su territorio. Tesis doctoral inédita, Universidad de Granada, 2011

- SÁNCHEZ LÓPEZ, E. (2014) El acueducto romano de Almuñécar. Análisis hidráulico. Madrider Mitteilungen, n. ${ }^{\circ}$ 55, 2014, pp. 379-397

- SÁNCHEZ LÓPEZ, E.; MORENO PÉREZ, S. (2012) El acueducto de Almuñécar. Revisión de su trazado y contextualización. Pyrenae, n. ${ }^{\circ}$ 43.1, 2012, pp. 83-106

- SÁNCHEZ LÓPEZ, E.; PÉREZ MARRERO, J.; ORFILA PONS, M. et ál. (2009) Nuevas consideraciones sobre el acueducto romano de Almuñécar. En HUERTA FERNÁNDEZ, S. (coord.) Actas del Sexto Congreso Nacional Historia de la construcción, vol. II. Valencia: Instituto Juan de Herrera, 2009, pp. 1297-1306

- SÁNCHEZ LÓPEZ, E.; PÉREZ MARRERO, J.; ORFILA PONS, M. et ál. (2010) El municipium de Sexi Firmum Iulium y el agua. En LAGÓSTENA BARRIOS, L. G.; CAÑIZAR PALACIOS, J. L.; PONS PUJOL, L. (ed.) Aquam perducendam curavit. Captación, uso y administración del agua en las ciudades de la Bética y el Occidente romano. Cádiz: Universidad, 2010, pp. 197-215

- tovar sabio, A.; CAMERO UCLES, T. (1986) Conducción romana de Sexi. Proyecto fin de carrera inédito, 1986

- YÁÑEZ JERÓNIMO, J. D.; GONZÁLEZ ALMENDROS, P.; BUSTOS PRETEL, A. (1994) Nuestra Señora la Virgen del Agua. "Jete". Granada: Caja Rural, 1994 